Proyecciones Journal of Mathematics Vol. 31, No 1, pp. 65-79, March 2012. Universidad Católica del Norte

Antofagasta - Chile

DOI: 10.4067/S0716-09172012000100007

\title{
Half-Sweep Geometric Mean Iterative Method for the Repeated Simpson Solution of Second Kind Linear Fredholm Integral Equations
}

\author{
MOHANA SUNDARAM MUTHUVALU \\ UNIVERSITI MALAYSIA SABAH, MALAYSIA \\ and \\ JUMAT SULAIMAN \\ UNIVERSITI MALAYSIA SABAH, MALAYSIA \\ Received: August 2010. Accepted : September 2011
}

\begin{abstract}
In previous studies, the effectiveness of the Half-Sweep Geometric Mean (HSGM) iterative method has been shown in solving first and second kind linear Fredholm integral equations using repeated trapezoidal (RT) discretization scheme. In this work, we investigate the efficiency of the HSGM method to solve dense linear system generated from the discretization of the second kind linear Fredholm integral equations by using repeated Simpson's $\frac{1}{3}$ (RS1) scheme. The formulation and implementation of the proposed method are also presented. In addition, several numerical simulations and computational complexity analysis were also included to verify the efficiency of the proposed method.
\end{abstract}

Keywords: Linear Fredholm equations, half-sweep iteration, repeated Simpson, Geometric Mean

Mathematics Subject Classification: 41A55, 45A05, 45B05, 65F10, 65 Y20 


\section{Introduction}

Integral equations of various types play an important role in many fields of science and engineering. On the other hand, integral equations are encountered in numerous applications in many fields including continuum mechanics, potential theory, geophysics, electricity and magnetism, kinetic theory of gases, hereditary phenomena in physics and biology, renewal theory, quantum mechanics, radiation, optimization, optimal control systems, communication theory, mathematical economics, population genetics, queuing theory, medicine, mathematical problems of radiative equilibrium, particle transport problems of astrophysics and reactor theory, acoustics, fluid mechanics, steady state heat conduction, fracture mechanics, and radiative heat transfer problems [25]. The most frequently investigated integral equations are Fredholm linear equation and its nonlinear counterparts. In this paper, linear Fredholm integral equations of the second kind are considered. Generally, second kind linear integral equations of Fredholm type in the generic form can be defined as follows

$$
\lambda y(x)-\int_{\Gamma} K(x, t) y(t) d t=f(x), \Gamma=[a, b], \lambda \neq 0
$$

where the parameter $\lambda$, kernel $K$ and free term $f$ are given, and $y$ is the unknown function to be determined. Kernel $K$ is called Fredholm kernel if the kernel in Eq. (1.1) is continuous on the square $S=\{a \leq x \leq b, a \leq$ $t \leq b\}$ or at least square integrable on this square and it is also assumed to be absolutely integrable and satisfy other properties that are sufficient to imply the Fredholm alternative theorem. Meanwhile, Eq. (1.1) also can be rewritten in the equivalent operator form

$$
(\lambda-\kappa) y=f
$$

where the integral operator can be defined as follows

$$
\kappa y(t)=\int_{\Gamma} K(x, t) y(t) d t .
$$

Theorem (Fredholm Alternative) [4]

Let $\chi$ be a Banach space and let $\kappa: \chi \rightarrow \chi$ be compact. Then the equation $(\lambda-\kappa) y=f, \lambda \neq 0$ has a unique solution $x \in \chi$ if and only if the homogeneous equation $(\lambda-\kappa) z=0$ has only the trivial solution $z=0$. In such a case, the operator $\lambda-\kappa: \chi \underset{\text { onto }}{\stackrel{1-1}{\longrightarrow}} \chi$ has a bounded inverse $(\lambda-\kappa)^{-1}$. 
Definition (Compact operators) [4]

Let $\chi$ and $Y$ be normed vector space and let $\kappa: \chi \rightarrow Y$ be linear. Then $\kappa$ is compact if the set $\{\kappa x \mid\|x\| x \leq 1\}$ has compact closure in $Y$. This is equivalent to saying that for every bounded sequence $\left\{x_{n}\right\} \subset \chi$, the sequences $\left\{\kappa x_{n}\right\}$ have a subsequence that is convergent to some points in $Y$. Compact operators are also called completely continuous operators.

A numerical approach to the solution of integral equations is an essential branch of scientific inquiry. As a matter of fact, some valid methods of solving linear Fredholm integral equations have been developed in recent years. To solve Eq. (1.1) numerically, we either seek to determine an approximate solution by using the quadrature method $[8,11,12,14,15,20]$, or use the projection method $[5,6,9,10]$. Such discretizations of integral equations lead to dense linear systems and can be prohibitively expensive to solve using direct methods as the order of the linear system increases. Thus, iterative methods are the natural options for efficient solutions.

Consequently, the concept of the two-stage iterative method has been proposed widely to be one of the efficient methods for solving any linear system. The two-stage iterative method, which is also called as inner/outer iterative scheme was introduced by Nichols [17]. Actually, there are many two-stage iterative methods can be considered such as Alternating Group Explicit (AGE) [7], Iterative Alternating Decomposition Explicit (IADE) [21], Reduced Iterative Alternating Decomposition Explicit (RIADE) [22], Block Jacobi [3] and Arithmetic Mean (AM) [19] methods.

Standard AM method also named as the Full-Sweep Arithmetic Mean (FSAM) method has been modified by combining the concept of half-sweep iteration and FSAM method, and then called as the Half-Sweep Arithmetic Mean (HSAM) method [23]. The concept of the half-sweep iteration method is introduced via the Explicit Decoupled Group (EDG) iterative method for solving two-dimensional Poisson equations [1]. Apart from the AM iterative methods, another two-stage method that is Half-Sweep Geometric Mean (HSGM) method has been proposed [24]. HSGM method is the combination of the half-sweep iteration and Full-Sweep Geometric Mean (FSGM) method. Further studies to verify the effectiveness of the HSGM method with Crank-Nicolson finite difference [2] and quadrature $[13,14]$ schemes to solve water quality model and linear Fredholm integral equations respectively have been carried out. However, in this paper, the application of the HSGM method using the half-sweep quadrature approximation equation based on repeated Simpson's $\frac{1}{3}$ (RS1) scheme for solving second kind linear Fredholm integral equations is examined. 
The outline of this paper is organized in following way. In Section 2, the formulation of the full- and half-sweep quadrature approximation equations will be explained. The latter section of this paper will discuss the formulations of the FSGM and HSGM methods, and some numerical results will be shown in fourth section to assert the performance of the proposed method. Besides that, analysis on computational complexity is mentioned in Section 5 and the concluding remarks are given in final section.

\section{Full- and Half-Sweep Quadrature Approximation Equa- tions}

As afore-mentioned, a discretization scheme based on method of quadrature was used to construct approximation equations for problem (1.1) by replacing the integral to finite sums. Generally, quadrature method can be defined as follows

$$
\int_{a}^{b} y(t) d t=\sum_{j=0}^{n} A_{j} y\left(t_{j}\right)+\epsilon_{n}(y)
$$

where $t_{j}(j=0,1,2, \cdots, n)$ is the abscissas of the partition points of the integration interval $[a, b], A_{j}(j=0,1,2, \cdots, n)$ is numerical coefficients that do not depend on the function $y(t)$ and $\epsilon_{n}(y)$ is the truncation error of Eq. (2.1). In order to facilitate the formulating of the full- and half-sweep quadrature approximation equations for problem (1.1), further discussion will be restricted onto RS1 scheme, which is based on quadratic polynomial interpolation formula with equally spaced data.

Meanwhile, Figure 2.1 shows the finite grid networks in order to form the full- and half-sweep repeated Simpson's $\frac{1}{3}$ approximation equations. 


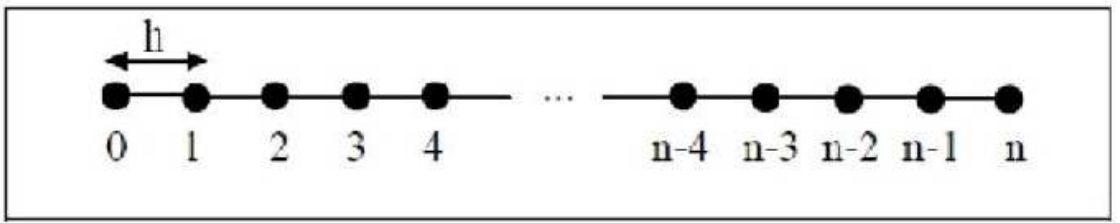

a)

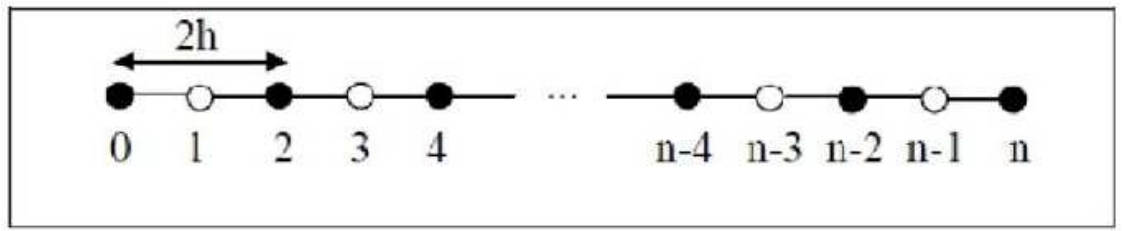

b)

Figure 2.1: a) and b) show distribution of uniform node points for the full- and half-sweep cases respectively

Based on Figure 2.1, the full and half-sweep iterative method will compute approximate values onto node points of type $\bullet$ only until the convergence criterion is reached. Then, other approximate solutions at remaining points (points of the different type, o) can be computed by using direct method $[16,20]$. In this paper, second order Lagrange interpolation method [16] will be used to compute the remaining points. Formulations to compute the remaining points using second order Lagrange interpolation for halfsweep iteration is defined as follows

$$
y= \begin{cases}\frac{3}{8} y_{i-1}+\frac{3}{4} y_{i+1}-\frac{1}{8} y_{i+3} & , \quad i=1,3,5, \cdots, n-3 \\ \frac{3}{4} y_{i-1}+\frac{3}{8} y_{i+1}-\frac{1}{8} y_{i-3} & , \quad i=n-1\end{cases}
$$

By applying Eq. (2.1) into Eq. (1.1) and neglecting the error, $\epsilon_{n}(y)$, a linear system can be formed for approximation values of $y(t)$. The following linear system generated using RS1 scheme can be easily shown in matrix form as 
follows

$$
M \underline{y}=\underline{f}
$$

where

$$
\begin{aligned}
& M=\left[\begin{array}{ccccc}
\lambda-A_{0} K_{0,0} & -A_{p} K_{0, p} & -A_{2 p} K_{0,2 p} & \cdots & -A_{n} K_{0, n} \\
-A_{0} K_{p, 0} & \lambda-A_{p} K_{p, p} & -A_{2 p} K_{p, 2 p} & \cdots & -A_{n} K_{p, n} \\
-A_{0} K_{2 p, 0} & -A_{p} K_{2 p, p} & \lambda-A_{2 p} K_{2 p, 2 p} & \cdots & -A_{n} K_{2 p, n} \\
\vdots & \vdots & \vdots & \ddots & \vdots \\
-A_{0} K_{n, 0} & -A_{p} K_{n, p} & -A_{2 p} K_{n, 2 p} & \cdots & \lambda-A_{n} K_{n, n}
\end{array}\right]_{\left(\frac{n}{p}+1\right) \times\left(\frac{n}{p}+1\right)} \\
& \underline{y}=\left[\begin{array}{lllllll}
y_{0} & y_{p} & y_{2 p} & \cdots & y_{n-2 p} & y_{n-p} & y_{n}
\end{array}\right]^{T},
\end{aligned}
$$

and

$$
\underline{f}=\left[\begin{array}{lllllll}
f_{0} & f_{p} & f_{2 p} & \cdots & f_{n-2 p} & f_{n-p} & f_{n}
\end{array}\right]^{T} .
$$

Based on RS1 scheme, numerical coefficient $A_{j}$ satisfy the following relations

$$
A_{j}= \begin{cases}\frac{1}{3} p h \quad, \quad j=0, n \\ \frac{4}{3} p h \quad, \quad j=p, 3 p, 5 p, \cdots, n-p \\ \frac{2}{3} p h \quad, \quad \text { otherwise }\end{cases}
$$

where the constant step size, $h$ is defined as follows

$$
h=\frac{b-a}{n}
$$

and $n$ is the number of subintervals in the interval $[a, b]$. The value of $p$, which corresponds to 1 and 2, represents the full- and half-sweep cases respectively. 


\section{Geometric Mean Iterative Methods}

As stated in previous section, FSGM and HSGM methods are one of the two-stage iterative methods and the iterative process of the methods involves of solving two independent systems such as $\underline{y}^{1}$ and $\underline{y}^{2}$. To develop the formulation of GM methods, express the coefficient matrix, $M$ as the matrix sum

$$
M=L+D+U
$$

where $L, D$ and $U$ are the strictly lower triangular, diagonal and strictly upper triangular matrices respectively. Thus, by adding positive acceleration parameter, $\omega$ the general scheme for FSGM and HSGM methods is defined as follows

$$
\left\{\begin{aligned}
(D+\omega L) \underline{y}^{1} & =[(1-\omega) D-\omega U] \underline{y}^{(k)}+\omega \underline{f} \\
(D+\omega U) \underline{y}^{2} & =[(1-\omega) D-\omega L] \underline{y}^{(k)}+\omega \underline{f} \\
\underline{y}^{(k+1)} & =\left(\underline{y}^{1} \circ \underline{y}^{2}\right)^{\frac{1}{2}}
\end{aligned}\right.
$$

where $\underline{y}^{(k)}$, $\circ$ and $(.)^{\frac{1}{2}}$ denote an unknown vector at the $k^{\text {th }}$ iteration, Hadamard product and Hadamard power respectively.

Practically, the value of $\omega$ will be determined by implementing some computer programs and then choose one value of $\omega$, where its number of iterations is the smallest. By determining values of matrices $L, D$ and $U$ as stated in Eq. (3.1), the general algorithm for FSGM and HSGM iterative methods using RS1 scheme to solve problem (1.1) would be generally described in Algorithm 1. The FSGM and HSGM algorithms are explicitly performed by using all equations at level (1) and (2) alternatively until the specified convergence criterion is satisfied.

Algorithm 1 FSGM and HSGM algorithms

i) Level (1)

for $i=0, p, 2 p, \cdots, n-2 p, n-p, n$ and $j=0, p, 2 p, \cdots, n-2 p, n-p, n$

Calculate

$$
y_{i}^{1} \leftarrow \begin{cases}(1-\omega) y_{i}^{(k)}+\frac{\omega\left(\sum_{j=p}^{n} A_{j} K_{i, j} y_{j}^{(k)}+f_{i}\right)}{\lambda-A_{i} K_{i, i}} & , i=0 \\ (1-\omega) y_{i}^{(k)}+\frac{\omega\left(\sum_{j=0}^{n-p} A_{j} K_{i, j} y_{j}^{1}+f_{i}\right)}{\lambda-A_{i} K_{i, i}} & , i=n \\ (1-\omega) y_{i}^{(k)}+\frac{\omega\left(\sum_{j=0}^{i-p} A_{j} K_{i, j} y_{j}^{1}+\sum_{j=i+p}^{n} A_{j} K_{i, j} y_{j}^{(k)}+f_{i}\right)}{\lambda-A_{i} K_{i, i}} & , \text { others }\end{cases}
$$

ii) Level (2) 
for $i=n, n-p, n-2 p, \cdots, 2 p, p, 0$ and $j=0, p, 2 p, \cdots, n-2 p, n-p, n$ Calculate

$$
y_{i}^{2} \leftarrow \begin{cases}(1-\omega) y_{i}^{(k)}+\frac{\omega\left(\sum_{j=p}^{n} A_{j} K_{i, j} y_{j}^{2}+f_{i}\right)}{\lambda-A_{i} K_{i, i}} & , i=0 \\ (1-\omega) y_{i}^{(k)}+\frac{\omega\left(\sum_{j=0}^{n-p} A_{j} K_{i, j} y_{j}^{(k)}+f_{i}\right)}{\lambda-A_{i} K_{i, i}} & , i=n \\ (1-\omega) y_{i}^{(k)}+\frac{\omega\left(\sum_{j=0}^{i-p} A_{j} K_{i, j} y_{j}^{(k)}+\sum_{j=i+p}^{n} A_{j} K_{i, j} y_{j}^{2}+f_{i}\right)}{\lambda-A_{i} K_{i, i}} & , \text { others }\end{cases}
$$

iii) for $i=0, p, 2 p, \cdots, n-2 p, n-p, n$ and $j=0, p, 2 p, \cdots, n-2 p, n-p, n$

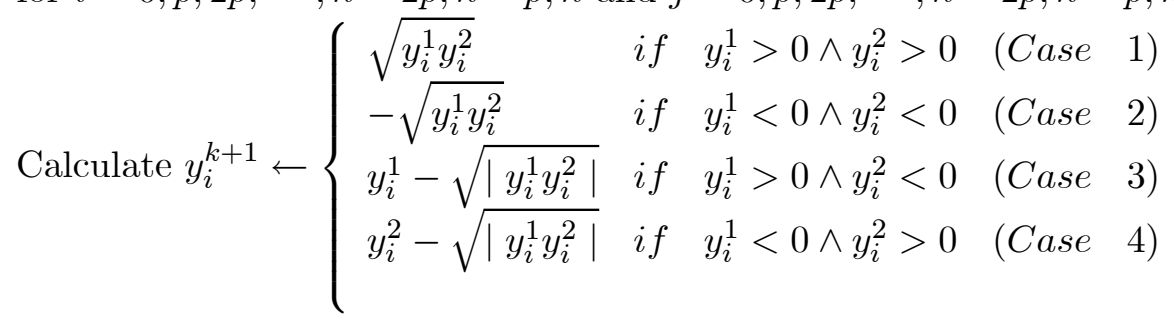

\section{Numerical Simulations}

In order to compare the performances of the iterative methods described in the previous section, several experiments were carried out on the following Fredholm integral equations problems.

Example 1 [25]

$$
y(x)-\int_{0}^{1}\left(4 x t-x^{2}\right) y(t) d t=x, 0 \leq x \leq 1
$$

and the exact solution is given by

$$
y(x)=24 x-9 x^{2} .
$$

Example 2 [18]

$$
y(x)-\int_{0}^{1}\left(x^{2}+t^{2}\right) y(t) d t=x^{6}-5 x^{3}+x+10,0 \leq x \leq 1
$$

with the exact solution

$$
y(x)=x^{6}-5 x^{3}+\frac{1045}{28} x^{2}+x+\frac{2141}{84} .
$$


Table 4.1: Comparison of a number of iterations, execution time (in seconds) and maximum absolute error for the iterative methods (Example 1)

\begin{tabular}{cccccc}
\hline \multirow{5}{*}{ Methods } & \multicolumn{5}{c}{ Number of iterations } \\
\cline { 2 - 6 } & $\mathbf{4 8 0}$ & $\mathbf{9 6 0}$ & $\mathbf{1 9 2 0}$ & $\mathbf{3 8 4 0}$ & $\mathbf{7 6 8 0}$ \\
\hline GS & 194 & 194 & 195 & 195 & 195 \\
FSGM & 83 & 83 & 83 & 83 & 83 \\
HSGM & 83 & 83 & 83 & 83 & 83 \\
\hline \multicolumn{5}{c}{ Execution time (in seconds) } \\
\hline Methods & $\mathbf{5}$ & $n$ \\
\cline { 2 - 6 } & $\mathbf{4 8 0}$ & $\mathbf{9 6 0}$ & $\mathbf{1 9 2 0}$ & $\mathbf{3 8 4 0}$ & $\mathbf{7 6 8 0}$ \\
\hline GS & 12.15 & 40.14 & 157.77 & 565.93 & 1104.18 \\
FSGM & 8.40 & 30.45 & 118.48 & 421.05 & 742.13 \\
HSGM & 3.44 & 9.56 & 35.39 & 147.08 & 556.82 \\
\hline \multicolumn{5}{c}{ Maximum absolute error } \\
\hline Methods & $\mathbf{5}$ & $n$ \\
\cline { 2 - 6 } & $\mathbf{4 8 0}$ & $\mathbf{9 6 0}$ & $\mathbf{1 9 2 0}$ \\
GS & $7.1564 \mathrm{E}-10$ & $7.5523 \mathrm{E}-10$ & $6.8680 \mathrm{E}-10$ & $6.9612 \mathrm{E}-10$ & $7.0084 \mathrm{E}-10$ \\
FSGM & $1.5996 \mathrm{E}-10$ & $1.6224 \mathrm{E}-10$ & $1.6341 \mathrm{E}-10$ & $1.6400 \mathrm{E}-10$ & $1.6422 \mathrm{E}-10$ \\
HSGM & $1.5647 \mathrm{E}-10$ & $1.6056 \mathrm{E}-10$ & $1.6259 \mathrm{E}-10$ & $1.6359 \mathrm{E}-10$ & $1.6410 \mathrm{E}-10$ \\
\hline
\end{tabular}

For comparison, the Gauss-Seidel (GS) method acts as the comparison control of numerical results. There are three parameters considered in numerical comparison such as number of iterations, execution time and maximum absolute error. Throughout the simulations, the convergence test considered the tolerance error, $\epsilon=10^{-10}$ and carried out on several different values of $n$. Meanwhile, the experimental values of $\omega$ were obtained within \pm 0.01 by running the program for different values of and choosing the one(s) that gives the minimum number of iterations. All the simulations were implemented by a computer with processor Intel(R) Core(TM) $2 \mathrm{CPU}$ $1.66 \mathrm{GHz}$ and computer codes were written in $\mathrm{C}$ programming language.

Results of numerical simulations, which were obtained from implementations of the GS, FSGM and HSGM iterative methods for Examples 1 and 2, have been recorded in Tables 4.1 and 4.2 respectively. Meanwhile, reduction percentages of the number of iterations and execution time for the FSGM and HSGM methods compared with GS method have been summarized in Table 4.3. 
Table 4.2: Comparison of a number of iterations, execution time (in seconds) and maximum absolute error for the iterative methods (Example 2)

\begin{tabular}{|c|c|c|c|c|c|}
\hline \multicolumn{6}{|c|}{ Number of iterations } \\
\hline \multirow[t]{2}{*}{ Methods } & \multicolumn{5}{|c|}{$n$} \\
\hline & 480 & 960 & 1920 & 3840 & 7680 \\
\hline GS & 56 & 56 & 56 & 56 & 56 \\
\hline FSGM & 32 & 32 & 32 & 32 & 32 \\
\hline HSGM & 32 & 32 & 32 & 32 & 32 \\
\hline \multicolumn{6}{|c|}{ Execution time (in seconds) } \\
\hline \multirow[t]{2}{*}{ Methods } & \multicolumn{5}{|c|}{$n$} \\
\hline & 480 & 960 & 1920 & 3840 & 7680 \\
\hline GS & 4.14 & 16.09 & 59.65 & 175.63 & 364.52 \\
\hline FSGM & 3.62 & 13.48 & 50.22 & 132.66 & 280.64 \\
\hline HSGM & 1.67 & 4.29 & 18.28 & 80.35 & 195.84 \\
\hline \multicolumn{6}{|c|}{ Maximum absolute error } \\
\hline \multirow[t]{2}{*}{ Methods } & \multicolumn{5}{|c|}{$n$} \\
\hline & 480 & 960 & 1920 & 3840 & 7680 \\
\hline GS & $5.8823 \mathrm{E}-10$ & $8.3052 \mathrm{E}-10$ & $1.2601 \mathrm{E}-10$ & $1.3006 \mathrm{E}-10$ & $1.3088 \mathrm{E}-1 \mathrm{C}$ \\
\hline FSGM & $6.3153 \mathrm{E}-10$ & 2.9677E-11 & $5.8409 \mathrm{E}-11$ & $6.1351 \mathrm{E}-11$ & $6.1606 \mathrm{E}-11$ \\
\hline HSGM & $4.1226 \mathrm{E}-9$ & $5.1123 \mathrm{E}-10$ & $6.3440 \mathrm{E}-11$ & $8.5116 \mathrm{E}-11$ & $1.6050 \mathrm{E}-11$ \\
\hline
\end{tabular}

Table 4.3: Reduction percentages of the number of iterations and execution time for the FSGM and HSGM methods compared with GS method

\begin{tabular}{ccc}
\hline \multicolumn{3}{c}{ Number of iterations } \\
\hline Methods & Example 1 & Example 2 \\
\hline FSGM & $57.21-57.44 \%$ & $42.85-42.86 \%$ \\
HSGM & $57.21-57.44 \%$ & $42.85-42.86 \%$ \\
\hline \multicolumn{3}{c}{ Execution time } \\
\hline Methods & Example 1 & Example 2 \\
\hline FSGM & $24.14-32.79 \%$ & $12.56-24.47 \%$ \\
HSGM & $49.57-77.57 \%$ & $46.27-73.34 \%$ \\
\hline
\end{tabular}


Table 5.1: Total computing operations involved for the FSGM and HSGM methods

\begin{tabular}{ccc}
\hline \multicolumn{3}{c}{ Total operations } \\
\hline Case & FSGM & HSGM \\
\hline Case 1 & $\left(6 n^{2}+22 n+16\right) k$ & $\left(\frac{3}{2} n^{2}+11 n+16\right) k+4 n$ \\
Case 2 & $\left(6 n^{2}+23 n+17\right) k$ & $\left(\frac{3}{2} n^{2}+\frac{23}{2} n+17\right) k+4 n$ \\
Case 3 & $\left(6 n^{2}+24 n+18\right) k$ & $\left(\frac{3}{2} n^{2}+12 n+18\right) k+4 n$ \\
Case 4 & $\left(6 n^{2}+24 n+18\right) k$ & $\left(\frac{3}{2} n^{2}+12 n+18\right) k+4 n$ \\
\hline
\end{tabular}

\section{Computational Complexity Analysis}

In order to measure the computational complexity of the FSGM and HSGM methods, an estimation amount of the computational work required for iterative methods have been conducted. The computational work is estimated by considering the arithmetic operations performed per iteration. Based on Algorithm 1, it can be observed that there are four different cases for both GM methods. In estimating the computational work for GM iterative methods, the value for kernel $K$, function $f$ and numerical coefficient $A_{j}$ are store beforehand. Assuming that the execution times for the operations addition, subtraction, multiplication, division and square root are roughly the same, the total arithmetic operations involved for FSGM and HSGM methods are summarized in Table 5.1.

\section{Conclusions}

In this paper, we present an application of the HSGM iterative method for solving dense linear systems arising from the discretization of the second kind linear Fredholm integral equations by using repeated Simpson's $\frac{1}{3}$ scheme. Through numerical results obtained for Examples 1 and 2, it clearly shows that by applying the GM methods can reduce number of iterations and execution time compared to the GS method. At the same time, it has been shown that, applying the half-sweep iterations reduces the computational time in the implementation of the iterative method. This is mainly because of computational complexity of the HSGM method which is approximately $75 \%$ less than FSGM method, since the implementation of half-sweep iteration will only consider half of all interior node points in a solution domain. Overall, the numerical results show that the HSGM method is a better method compared to the GS and FSGM methods in the 
sense of number of iterations and execution time.

\section{References}

[1] Abdullah, A. R. The four point Explicit Decoupled Group (EDG) method: A fast Poisson solver. International Journal of Computer Mathematics 38: pp. 61-70, (1991).

[2] Abdullah, M. H., J. Sulaiman, A. Saudi, M. K. Hasan, and M. Othman. A numerical simulation on water quality model using Half-Sweep Geometric Mean method. Proceedings of the Second Southeast Asian Natural Resources and Environment Management Conference: pp. 2529, (2006).

[3] Allahviranloo, T., E. Ahmady, N. Ahmady, and K. S. Alketaby. Block Jacobi two-stage method with Gauss-Sidel inner iterations for fuzzy system of linear equations. Applied Mathematics and Computation 175: pp. 1217-1228, (2006).

[4] Atkinson, K. E. The Numerical Solution of Integral Equations of the Second Kind. Cambridge: Cambridge University Press. (1997).

[5] Cattani, C., and A. Kudreyko. Harmonic wavelet method towards solution of the Fredholm type integral equations of the second kind. Applied Mathematics and Computation 215: pp. 4164-4171, (2010).

[6] Chen, Z., B. Wu, and Y. Xu. Fast numerical collocation solutions of integral equations. Communications on Pure and Applied Analysis 6: pp. 643-666, (2007).

[7] Evans, D. J. The Alternating Group Explicit (AGE) matrix iterative method. Applied Mathematical Modelling 11: pp. 256-263, (1987).

[8] Kang, S. -Y., I. Koltracht, and G. Rawitscher. Nyström-ClenshawCurtis Quadrature for Integral Equations with Discontinuous Kernels. Mathematics of Computation 72: pp. 729-756, (2003).

[9] Liu, Y. Application of the Chebyshev polynomial in solving Fredholm integral equations. shape Mathematical and Computer Modelling 50: pp. 465-469, (2009). 
[10] Long, G., M. M. Sahani, and G. Nelakanti. Polynomially based multiprojection methods for Fredholm integral equations of the second kind. Applied Mathematics and Computation 215: pp. 147-155, (2009).

[11] Mastroianni, G., and G. Monegato. Truncated quadrature rules over and Nyström-type methods. SIAM Journal on Numerical Analysis 41: pp. 1870-1892, (2004).

[12] Mirzaee, F., and S. Piroozfar. (2010). Numerical solution of linear Fredholm integral equations via modified Simpson's quadrature rule. Journal of King Saud University - Science 23: (2011).

[13] Muthuvalu, M. S., and J. Sulaiman. Half-Sweep Geometric Mean method for solution of linear Fredholm equations. Matematika 24: pp. 75-84, (2008).

[14] Muthuvalu, M. S., and J. Sulaiman. Numerical solutions of second kind Fredholm integral equations using Half-Sweep Geometric Mean method. Proceedings of the IEEE International Symposium on Information Technology: pp. 1927-1934, (2008).

[15] Muthuvalu, M. S., and J. Sulaiman. Half-Sweep Arithmetic Mean method with high-order Newton-Cotes quadrature schemes to solve linear second kind Fredholm equations. Journal of Fundamental Sciences 5: pp. 7-16, (2009).

[16] Muthuvalu, M. S., and J. Sulaiman. Numerical solution of second kind linear Fredholm integral equations using QSGS iterative method with high-order Newton-Cotes quadrature schemes. Malaysian Journal of Mathematical Sciences 5: pp. 85-100, (2011).

[17] Nichols, N. K. On the convergence of two-stage iterative process for solving linear equations. SIAM Journal on Numerical Analysis 10: pp. 460-469, (1973).

[18] Polyanin, A. D., and A. V. Manzhirov. Handbook of Integral Equations. CRC Press LCC, (1998).

[19] Ruggiero, V., and E. Galligani. An iterative method for large sparse systems on a vector computer. Computers \& Mathematics with Applications 20: pp. 25-28, (1990). 
[20] Saberi-Nadjafi, J., and M. Heidari. Solving integral equations of the second kind with repeated modified trapezoid quadrature method. Applied Mathematics and Computation 189: pp. 980-985, (2007).

[21] Sahimi, M. S., A. Ahmad, and A. A. Bakar. The Iterative Alternating Decomposition Explicit (IADE) method to solve the heat conduction equation. International Journal of Computer Mathematics 47: pp. 219229, (1993).

[22] Sahimi, M. S., and M. Khatim. The Reduced Iterative Alternating Decomposition Explicit (RIADE) method for diffusion equation. Pertanika Journal of Science \& Technology 9: pp. 13-20, (2001).

[23] Sulaiman, J., M. Othman, and M. K. Hasan. A new Half-Sweep Arithmetic Mean (HSAM) algorithm for two-point boundary value problems. Proceedings of the International Conference on Statistics and Mathematics and Its Application in the Development of Science and Technology: pp. 169-173, (2004).

[24] Sulaiman, J., M. Othman, N. Yaacob, and M. K. Hasan. Half-Sweep Geometric Mean (HSGM) method using fourth-order finite difference scheme for two-point boundary problems. Proceedings of the First International Conference on Mathematics and Statistics: pp. 25-33, (2006).

[25] Wang, W. A new mechanical algorithm for solving the second kind of Fredholm integral equation. Applied Mathematics and Computation 172: pp. 946-962, (2006).

\section{Mohana Sundaram Muthuvalu}

School of Science and Technology,

Universiti Malaysia Sabah

Jalan UMS, 88400 Kota Kinabalu,

Sabah,

Malaysia

e-mail : sundaram_at2@yahoo.com

and 


\section{Jumat Sulaiman}

School of Science and Technology,

Universiti Malaysia Sabah

Jalan UMS, 88400 Kota Kinabalu,

Sabah,

Malaysia

e-mail : jumat@ums.edu.my 